\title{
Distortion correction by phase conjugation of nonparaxial vectorial beams: a general proof
}

\author{
Alessandro Ciattoni and Paolo Di Porto \\ Department of Physics, University of L'Aquila, 67010 L'Aquila, Italy, and \\ Istituto Nazionale Fisica della Materia, Unità di Roma "La Sapienza," Rome, Italy
}

Bruno Crosignani and Amnon Yariv

Department of Applied Physics, California Institute of Technology, Pasadena, California 91125

Received July 31, 2000

\begin{abstract}
We present a general proof of the distortion-correction theorem, that is, of the possibility of correcting wave distortion by the technique of optical phase conjugation. The proof is valid for fully vectorial nonparaxial propagation in the presence of a tensorial refractive-index perturbation and backscattering of the incident field. (C) 2001 Optical Society of America

OCIS codes: $190.5040,260.2110$.
\end{abstract}

The phase-conjugation process allows for the possibility of healing possible distortions introduced by the presence of the refractive index on a forward-propagating monochromatic electromagnetic beam. This compensation can be obtained by reflection of the beam by a phase-conjugate mirror, which produces a backward-propagating beam with a boundary condition that is the complex conjugate of the field that is incident upon the mirror. The proof presented in Ref. 1 hinges on the validity of the parabolic equation for the slowly varying amplitude, $\mathbf{A}(x, y, z)$, of the forward-propagating electric field, $\mathbf{E}(x, y, z ; t)=\mathbf{A}(x, y, z) \exp [i(k z-\omega t)]$, i.e.,

$$
\left(i \frac{\partial}{\partial z}+\frac{1}{2 k} \nabla_{\perp}^{2}\right) \mathbf{A}\left(\mathbf{r}_{\perp}, z\right)=-\frac{k}{n_{0}} \delta n(\mathbf{r}) \mathbf{A}\left(\mathbf{r}_{\perp}, z\right),
$$

where $\nabla_{\perp}^{2}=\partial^{2} / \partial x^{2}+\partial^{2} / \partial y^{2}, \mathbf{r}=(x, y, z)=\left(\mathbf{r}_{\perp}, z\right)$, $k=\omega n_{0} / c$, and $n=n_{0}+\delta n(\mathbf{r})$ is the refractive index of the medium. If we take the complex conjugate of Eq. (1), it is immediately clear that, if $\delta n$ is real, the complex conjugate amplitude $\mathbf{A}^{*}(x, y, z)$ obeys the same equation as the amplitude of the counterpropagating beam. As a consequence, if on a given plane $z=L$ we generate a backward-propagating wave that is the complex conjugate of $\mathbf{A}(x, y, z=L)$, the wave will propagate while remaining everywhere the complex conjugate of the forward-propagating field. Note that it is expedient for the validity of this argument that Eq. (1) contain $\partial / \partial z$ as a firstorder term so that knowledge of only the field on a given plane is completely sufficient to determine the solution of Eq. (1) at any other position. The price that one has to pay for this circumstance is that the proof suffers limitations owing to the fact that Eq. (1) is a scalar equation that is valid in the paraxial regime and does not account for coupling between forward- and backward-propagating fields. We wish to present in this Letter a very general, straightforward proof of the distortion-correction theorem for which it is, again, expedient that $\partial / \partial z$ appear in the propagation equations as a first-order term but that does not suffer the limitations discussed above. In this respect, it is interesting to recall a result that we can obtain by starting directly from Maxwell's equations (which are first order in $\partial / \partial z$ ). After writing the solutions in the form $\mathbf{E}(\mathbf{r}, t)=\mathbf{E}(\mathbf{r}) \exp (-i \omega t)$, $\mathbf{H}(\mathbf{r}, t)=\mathbf{H}(\mathbf{r}) \exp (-i \omega t)$ and introducing the field $\mathbf{E}^{\prime}(\mathbf{r})=\mathbf{E}^{*}(\mathbf{r}), \mathbf{H}^{\prime}(\mathbf{r})=-\mathbf{H}^{*}(\mathbf{r})$, which possesses everywhere a power flow $(1 / 2) \operatorname{Re}\left(\mathbf{E} \times \mathbf{H}^{*}\right)$ that is the negative of the power field of the original fields $\mathbf{E}$ and $\mathbf{H}$, we can show that $\mathbf{E}^{\prime}, \mathbf{H}^{\prime}$ obey, for a real refractive-index distribution, a set of equations identical to those obeyed by $\mathbf{E}(\mathbf{r})$ and $\mathbf{H}(\mathbf{r}){ }^{2}$ This argument, however, does not constitute a proof of the phase-conjugation technique. In fact, the proof requires, as in the proof in Ref. 1, that the propagation equation obeyed by the complex conjugate $\mathbf{E}^{+*}$ of the forward-propagating field obey the same equation as the backward-propagating field $\mathbf{E}^{-}$. However, fields $\mathbf{E}^{\prime}$ and $\mathbf{H}^{\prime}$ actually contain, in general in an indistinguishable way, both forward- and backwardpropagation fields.

A satisfactory theory of the phase-conjugation process based on the framework of Maxwell's electromagnetic theory was provided by Agarwal et al. ${ }^{3}$ It is based on the introduction of an integral equation formulation of propagation in the presence of a scalar refractive-index distribution whose solution is obtained by means of an iterative procedure that takes into account the contribution of higher-order terms in the perturbation expansion of the scattered field.

In the following, we present a straightforward general proof that is valid for vectorial nonparaxial propagation and that includes coupling between counterpropagating fields and the presence of a tensorial refractive-index perturbation. To this end, we recall that in Ref. 4 we investigated nonparaxial vectorial propagation of a monochromatic electromagnetic wave in a medium characterized by a real refractive-index distribution $\mathbf{n}=n_{0}+\delta \overleftrightarrow{\mathbf{n}}(\mathbf{r}), \delta \overleftrightarrow{\mathbf{n}}(\mathbf{r})$ being a tensorial perturbation over the hologeneous background associated with $n_{0}$. More precisely, after the electromagnetic field is expressed in terms of its transverse forward- and backward-propagating electric $\left[\mathbf{E}_{\perp}(\mathbf{r})=\mathbf{E}_{\perp}^{+}\left(\mathbf{r}_{\perp}, z\right)+\mathbf{E}_{\perp}^{-}\left(\mathbf{r}_{\perp}, z\right)\right]$ and magnetic $\left[\mathbf{B}_{\perp}(\mathbf{r})=\mathbf{B}_{\perp}^{+}\left(\mathbf{r}_{\perp}, z\right)+\mathbf{B}_{\perp}^{-}\left(\mathbf{r}_{\perp}, z\right)\right]$ components, the set of 
propagation equations that describe the evolution of $\mathbf{E}_{\perp}^{+}(\mathbf{r})$ and $\mathbf{E}_{\perp}^{-}(\mathbf{r})$ in an arbitrary $z$ direction reads as [see Eq. (E1) of Ref. 4]

$$
\begin{aligned}
& \left(i \frac{\partial}{\partial z} \pm \hat{T}\right) \mathbf{E}_{\perp}^{ \pm}\left(\mathbf{r}_{\perp}, z\right)=\mp\left(k^{2} / n_{0}\right) \hat{S}\left[\delta \overleftrightarrow{\mathbf{n}}_{\perp}:\left(\mathbf{E}_{\perp}^{+}+\mathbf{E}_{\perp}^{-}\right)\right] \\
& +\frac{1}{n_{0}} \nabla_{\perp}\left\{\delta n_{z z} \hat{S}\left[\nabla_{\perp} \cdot\left(\mathbf{E}_{\perp}^{+}-\mathbf{E}_{\perp}^{-}\right)\right]\right\} \mp \frac{1}{n_{0}} \hat{S} \nabla_{\perp}\left\{\nabla_{\perp}\right. \\
& \left.\cdot\left[\delta \overleftrightarrow{\mathbf{n}}_{\perp}:\left(\mathbf{E}_{\perp}^{+}+\mathbf{E}_{\perp}^{-}\right)\right]\right\}-\frac{i}{n_{0}} \nabla_{\perp}\left[\delta \mathbf{n}^{\prime} \cdot\left(\mathbf{E}_{\perp}^{+}+\mathbf{E}_{\perp}^{-}\right)\right] \mp \frac{i k}{n_{0}} \\
& \times \hat{S}\left\{\delta \mathbf{n}^{\prime} \hat{S}\left[\nabla_{\perp} \cdot\left(\mathbf{E}_{\perp}^{+}-\mathbf{E}_{\perp}^{-}\right)\right]\right\} \mp \frac{i}{n_{0}} \hat{S} \nabla_{\perp}\left(\nabla _ { \perp } \cdot \left\{\delta \mathbf { n } ^ { \prime } \hat { S } \left[\nabla_{\perp}\right.\right.\right. \\
& \left.\left.\left.\cdot\left(\mathbf{E}_{\perp}^{+}-\mathbf{E}_{\perp}^{-}\right)\right]\right\}\right),
\end{aligned}
$$

where $\delta \overleftrightarrow{\mathbf{n}}_{\perp}$ is the transverse part of the tensor $\delta \overleftrightarrow{\mathbf{n}}(\mathbf{r})$, $\delta \mathbf{n}^{\prime}=\delta n_{z x} \hat{\mathbf{x}}+\delta n_{z y} \hat{\mathbf{y}}$, and the operators $\hat{T}=\left(k^{2}+\right.$ $\left.\nabla_{\perp}^{2}\right)^{1 / 2}$ and $\hat{S}=1 /\left(k^{2}+\nabla_{\perp}^{2}\right)^{1 / 2}$ can be given a precise meaning in connection with the transverse-space Fourier transforms $\tilde{\mathbf{E}}_{\perp}^{ \pm}\left(\mathbf{k}_{\perp}, z\right)$ of $\mathbf{E}_{\perp}^{ \pm}\left(\mathbf{r}_{\perp}, z\right)$ through

$$
\begin{aligned}
\hat{T} \mathbf{E}_{\perp}^{ \pm}\left(\mathbf{r}_{\perp}, z\right)= & \int \mathrm{d}^{2} \mathbf{k}_{\perp}\left(k^{2}-k_{\perp}^{2}\right)^{1 / 2} \\
& \times \exp \left(i \mathbf{k}_{\perp} \cdot \mathbf{r}_{\perp}\right) \tilde{\mathbf{E}}_{\perp}^{ \pm}\left(\mathbf{k}_{\perp}, z\right), \\
\hat{S} \mathbf{E}_{\perp}^{ \pm}\left(\mathbf{r}_{\perp}, z\right)= & \int \mathrm{d}^{2} \mathbf{k}_{\perp}\left[1 /\left(k^{2}-k_{\perp}^{2}\right)\right]^{1 / 2} \\
& \times \exp \left(i \mathbf{k}_{\perp} \cdot \mathbf{r}_{\perp}\right) \tilde{\mathbf{E}}_{\perp}^{ \pm}\left(\mathbf{k}_{\perp}, z\right) .
\end{aligned}
$$

By taking the complex conjugate of Eq. (2) and observing that, in the absence of evanescent modes $\left(k_{\perp}<\right.$ $k$ ), both $\hat{T}$ and $\hat{S}$ remain the same under this operation, we obtain

$$
\begin{aligned}
& \left(i \frac{\partial}{\partial z} \mp \hat{T}\right) \mathbf{E}_{\perp}^{土^{*}}\left(\mathbf{r}_{\perp}, z\right)= \pm\left(k^{2} / n_{0}\right) \hat{S}\left[\delta \overleftrightarrow{\mathbf{n}}_{\perp}:\left(\mathbf{E}_{\perp}^{*^{*}}+\mathbf{E}_{\perp}^{*^{*}}\right)\right] \\
& -\frac{1}{n_{0}} \nabla_{\perp}\left\{\delta n_{z z} \hat{S}\left[\nabla_{\perp} \cdot\left(\mathbf{E}_{\perp}^{+*}-\mathbf{E}_{\perp}^{-*}\right)\right]\right\} \pm \frac{1}{n_{0}} \hat{S} \nabla_{\perp}\left\{\nabla_{\perp}\right. \\
& \left.\cdot\left[\delta \overleftrightarrow{\mathbf{n}}_{\perp}:\left(\mathbf{E}_{\perp}^{+*}+\mathbf{E}_{\perp}^{-*}\right)\right]\right\}-\frac{i}{n_{0}} \nabla_{\perp}\left[\delta \mathbf{n}^{\prime} \cdot\left(\mathbf{E}_{\perp}^{+*}+\mathbf{E}_{\perp}^{-*}\right)\right] \mp \frac{i k}{n_{0}} \\
& \times \hat{S}\left\{\delta \mathbf{n}^{\prime} \hat{S}\left[\nabla_{\perp} \cdot\left(\mathbf{E}_{\perp}^{+*}-\mathbf{E}_{\perp}^{*^{*}}\right)\right]\right\} \mp \frac{i}{n_{0}} \hat{S} \nabla_{\perp}\left(\nabla _ { \perp } \cdot \left\{\delta \mathbf { n } ^ { \prime } \hat { S } \left[\nabla_{\perp}\right.\right.\right. \\
& \left.\left.\left.\cdot\left(\mathbf{E}_{\perp}^{+^{*}}-\mathbf{E}_{\perp}^{*^{*}}\right)\right]\right\}\right) .
\end{aligned}
$$

It is found straightforwardly that Eqs. (2) and (4) transform one into the other through the exchange $\mathbf{E}_{\perp}^{ \pm *}\left(\mathbf{r}_{\perp}, z\right) \leftrightarrow \mathbf{E}_{\perp}^{\mp}\left(\mathbf{r}_{\perp}, z\right)$, which expresses the phaseconjugation condition. In fact, the set of Eqs. (2) and (4) is first order in $\partial / \partial z$, and thus knowledge of $\mathbf{E}_{\perp}^{ \pm}\left(\mathbf{E}_{\perp}^{ \pm}{ }^{*}\right)$ at two given planes, $z=z_{1}$ and $z=z_{2}$, is sufficient for specifying the solution of this set of equations. Thus, if one produces, by means of a phase-conjugate mirror on a plane $z=L$ the condition $\mathbf{E}_{\perp}^{-}\left(\mathbf{r}_{\perp}, z=L\right)=\mu \mathbf{E}_{\perp}^{+*}\left(\mathbf{r}_{\perp}, z=L\right)$, where $\mu$ represents the mirror reflectivity, the backward-propagating beam is healed by the possible distortion introduced by the presence of the refractive medium and regains its original state. Accordingly, if the refractive index is real and evanescent modes are negligible, the phase-conjugation technique applies to the general case of optical vectorial nonparaxial propagation in the presence of a tensorial refractive-index perturbation and coupling between counterpropagating waves.

Finally, we observe that, in the case of random fields, phase conjugation of partially coherent waves can be investigated by study of their correlation functions, such as the spectral densities $\left\langle\mathbf{E}_{\perp}^{ \pm}\left(\mathbf{r}_{\perp}, z\right) \mathbf{E}_{\perp}^{ \pm}{ }^{*}\left(\mathbf{r}_{\perp}^{\prime}, z\right)\right\rangle .^{5}$ In this context we can derive a closed set of equations for these quantities from Eqs. (2) and (4) by relying on the standard formalism that describes propagation of the cross-spectral density. ${ }^{6}$

This work was supported by U.S. Army Research Office contract DAAH04-96-1-0389 and National Science Foundation contract ECS-9632937. B. Crosignani's e-mail address is bruno.crosignani@aquila.infn.it.

\section{References}

1. A. Yariv, IEEE J. Quantum Electron. QE-14, 650 (1978).

2. See, e.g., A. Yariv, Quantum Electronics, 3rd ed. (Wiley, New York, 1989).

3. G. S. Agarwal, A. T. Friberg, and E. Wolf, J. Opt. Soc. Am. 73, 529 (1983).

4. A. Ciattoni, P. Di Porto, B. Crosignani, and A. Yariv, J. Opt. Soc. Am. B 17, 809 (2000).

5. G. Gbur and E. Wolf, Opt. Lett. 24, 10 (1999).

6. M. Born and E. Wolf, Principles of Optics, 7th ed. (Pergamon, Oxford, 1999). 